\title{
Cerebrospinal fluid volume change in neonates, infants, children, and adolescence - a magnetic resonance imaging study
}

Jin-Tae Kim, Young-Eun Jang, Joon-Hee Lee

Department of Anesthesiology and Pain Medicine, Seoul National University Hospital, Seoul, Seoul National University, College of Medicine, Republic of Korea

Background: The volume of cerebrospinal fluid (CSF) is the most important factor determining spinal anaesthesia level. However, the reference values for CSF volume have not been clearly determined in paediatric patients. In this study, we reviewed magnetic resonance (MR) images of paediatric patients of all ages, from neonates to adolescents, to provide a reference range of CSF volumes based on patient age, height, and weight.

Methods: Thoracolumbosacral (TLS)-CSF $(n=248)$ and lumbosacral (LS)-CSF volumes $(n=418)$ were measured from MR images obtained from paediatric patients. The correlation between TLS-CSF and age, height, and weight; and the correlation between LS-CSF and age, height, and weight were analysed.
Results: The TLS- and LS-CSF volumes showed a linear correlation with height $\left(r^{2}=0.661\right.$ and $r^{2}=0.730$, respectively), but a curvilinear correlation with age $\left(r^{2}=0.717\right.$ and $r^{2}=0.752$, respectively) and weight $\left(r^{2}=0.734\right.$ and $r^{2}=0.734$, respectively). The TLS- and LS-CSF volume/weight decreased with age and weight. The TLS-CSF volume was estimated to be $1.9 \mathrm{~mL} / \mathrm{kg}$, $1.6 \mathrm{~mL} / \mathrm{kg}$, and $1.0 \mathrm{~mL} / \mathrm{kg}$ in patients weighing $0-15 \mathrm{~kg}, 15-30$ $\mathrm{kg}$, and more than $30 \mathrm{~kg}$, respectively; and $1.9 \mathrm{~mL} / \mathrm{kg}, 1.6$ $\mathrm{mL} / \mathrm{kg}$, and $1.2 \mathrm{~mL} / \mathrm{kg}$ in patients aged $0-4$ years, $4-8$ years, and older than 8 years, respectively. The LSCSF volume was estimated to be half of the TLS-CSF volume.

Conclusions: When intrathecal dosage is determined for paediatric patients, the TLS- and LS-CSF volumes, which vary with weight, age, and height, should be considered.

Table. Patients' characteristics and the LS-CSF volume related parameters based on the 4 weight groups and 4 age groups

\begin{tabular}{|c|c|c|c|c|c|c|c|c|c|}
\hline & \multirow{2}{*}{ All $(n=418)$} & \multicolumn{4}{|c|}{ Weight groups } & \multicolumn{4}{|c|}{ Age groups } \\
\hline & & $0-10 \mathrm{~kg}(\mathrm{n}=77)$ & $10-15 \mathrm{~kg}(\mathrm{n}=101)$ & $15-30 \mathrm{~kg}(\mathrm{n}=147)$ & $>30 \mathrm{~kg}(\mathrm{n}=93)$ & $0-1$ years $(n=73)$ & $1-4$ years $(n=105)$ & 4-8 years $(n=119)$ & $>8$ years $(n=121)$ \\
\hline Age (month) ${ }^{\star}$ & $\begin{array}{l}70.5 \pm 57.3 \\
(0.0-221.0) \\
{[65.0-76.0]} \\
\end{array}$ & $\begin{array}{l}8.6 \pm 9.8 \\
(0.0-70.0) \\
{[6.4-10.8]} \\
\end{array}$ & $\begin{array}{c}30.8 \pm 17.4 \\
(7.0-87.0) \\
{[27.4-34.3]} \\
\end{array}$ & $\begin{array}{c}76.1 \pm 27.6 \\
(19.0-173.0) \\
{[71.6-80.6]} \\
\end{array}$ & $\begin{array}{c}156.0 \pm 30.0 \\
(82.0-221.0) \\
{[149.9-162.2]} \\
\end{array}$ & $\begin{array}{c}5.9 \pm 4.0 \\
(0.0-12.0) \\
{[4.9-6.8]} \\
\end{array}$ & $\begin{array}{l}28.3 \pm 10.5 \\
(13.0-48.0) \\
{[26.2-30.3]} \\
\end{array}$ & $\begin{array}{l}68.8 \pm 13.5 \\
(49.0-95.0) \\
{[66.4-71.3]} \\
\end{array}$ & $\begin{array}{c}147.2 \pm 31.0 \\
(97.0-221.0) \\
{[142.2-153.5]}\end{array}$ \\
\hline Height $(\mathrm{cm})^{\star}$ & $\begin{array}{c}103.5 \pm 28.9 \\
(45.0-184.0) \\
{[100.1-106.8]}\end{array}$ & $\begin{array}{l}68.8 \pm 10.5 \\
(45.0-94.2) \\
{[65.9-71.6]} \\
\end{array}$ & $\begin{array}{c}88.9 \pm 7.8 \\
(73.4-107.0) \\
{[87.3-90.6]} \\
\end{array}$ & $\begin{array}{c}112.2 \pm 10.6 \\
(86.0-144.6) \\
{[110.1-114.3]}\end{array}$ & $\begin{array}{c}155.0 \pm 13.3 \\
(122.0-184.0) \\
{[151.0-159.1]} \\
\end{array}$ & $\begin{array}{c}67.5 \pm 9.7 \\
(45.0-83.5) \\
{[64.8-70.2]} \\
\end{array}$ & $\begin{array}{c}88.0 \pm 7.2 \\
(73.0-105.3) \\
{[86.4-89.5]} \\
\end{array}$ & $\begin{array}{c}109.6 \pm 8.0 \\
(94.0-131.8) \\
{[107.9-111.2]}\end{array}$ & $\begin{array}{c}150.3 \pm 14.7 \\
(119.6-184.0) \\
{[146.7-155.0]}\end{array}$ \\
\hline Weight $(\mathrm{kg})^{\star}$ & $\begin{array}{l}21.9 \pm 15.9 \\
(2.6-104.7) \\
{[20.4-23.5]} \\
\end{array}$ & $\begin{array}{c}7.2 \pm 2.2 \\
(2.6-10.0) \\
{[6.7-7.7]} \\
\end{array}$ & $\begin{array}{c}12.6 \pm 1.6 \\
(10.1-15.0) \\
{[12.3-12.9]} \\
\end{array}$ & $\begin{array}{c}20.7 \pm 3.9 \\
(15.2-30.0) \\
{[20.1-21.4]} \\
\end{array}$ & $\begin{array}{c}46.2 \pm 15.5 \\
(30.5-104.7) \\
{[43.0-49.4]} \\
\end{array}$ & $\begin{array}{c}7.4 \pm 2.6 \\
(2.6-12.4) \\
{[6.8-8.0]} \\
\end{array}$ & $\begin{array}{c}12.7 \pm 2.4 \\
(8.0-18.5) \\
{[12.2-13.1]} \\
\end{array}$ & $\begin{array}{r}19.5 \pm 4.3 \\
(10.0-41.2) \\
{[18.7-20.3]} \\
\end{array}$ & $\begin{array}{c}40.7 \pm 15.4 \\
(17.9-104.7) \\
{[38.2-44.1]} \\
\end{array}$ \\
\hline $\begin{array}{l}\text { LS-CSF volume } \\
\qquad(\mathrm{mL})^{\star}\end{array}$ & $\begin{array}{c}14.6 \pm 7.2 \\
(2.1-44.7) \\
{[14.0-15.4]} \\
\end{array}$ & $\begin{array}{c}6.6 \pm 3.0 \\
(2.1-15.6) \\
{[5.9-7.3]} \\
\end{array}$ & $\begin{array}{c}11.3 \pm 3.2 \\
(6.4-25.1) \\
{[10.7-11.9]} \\
\end{array}$ & $\begin{array}{c}16.1 \pm 4.5 \\
(6.1-35.3) \\
{[15.3-16.8]} \\
\end{array}$ & $\begin{array}{c}22.8 \pm 7.1 \\
(10.6-44.7) \\
{[21.3-24.3]} \\
\end{array}$ & $\begin{array}{c}6.2 \pm 2.4 \\
(2.1-12.2) \\
{[5.6-6.8]} \\
\end{array}$ & $\begin{array}{c}11.4 \pm 3.0 \\
(4.1-20.9) \\
{[10.8-11.9]} \\
\end{array}$ & $\begin{array}{c}15.0 \pm 3.7 \\
(7.0-25.1) \\
{[14.3-15.7]} \\
\end{array}$ & $\begin{array}{c}22.3 \pm 6.7 \\
(11.7-44.7) \\
{[21.1-23.5]} \\
\end{array}$ \\
\hline $\begin{array}{c}\text { LS-CSF volume } \\
\text { per weight } \\
(\mathrm{mL} / \mathrm{kg})^{\star}\end{array}$ & $\begin{array}{l}0.78 \pm 0.25 \\
(0.23-1.67) \\
{[0.75-0.80]} \\
\end{array}$ & $\begin{array}{c}0.91 \pm 0.24 \\
(0.45-1.60) \\
{[0.86-0.96]^{+}} \\
\end{array}$ & $\begin{array}{c}0.89 \pm 0.20 \\
(0.53-1.67) \\
{[0.86-0.93]^{+}} \\
\end{array}$ & $\begin{array}{l}0.79 \pm 0.21 \\
(0.31-1.35) \\
{[0.75-0.82]} \\
\end{array}$ & $\begin{array}{l}0.52 \pm 0.17 \\
(0.23-1.08) \\
{[0.48-0.55]} \\
\end{array}$ & $\begin{array}{c}0.85 \pm 0.19 \\
(0.46-1.42) \\
{[0.81-0.90]^{+, \neq}} \\
\end{array}$ & $\begin{array}{c}0.91 \pm 0.21 \\
(0.38-1.60) \\
{[0.87-0.95]^{+}} \\
\end{array}$ & $\begin{array}{c}0.80 \pm 0.23 \\
(0.26-1.67) \\
{[0.75-0.84]^{\ddagger}} \\
\end{array}$ & $\begin{array}{l}0.60 \pm 0.23 \\
(0.23-1.35) \\
{[0.56-0.64]} \\
\end{array}$ \\
\hline
\end{tabular}

Data are presented as mean \pm standard deviation (range) [95\% confidence interval], LS-CSF: lumbosacral cerebrospinal fluid, ${ }^{*} P<0.05$ from repeated measured ANOVA, $\dagger$, $\ddagger$ No difference between each other

Figure 1. (A) The relationship between TLS-CSF volume and age, height, and weight; (B) The relationship between LS-CSF volume and age, height, and weight.
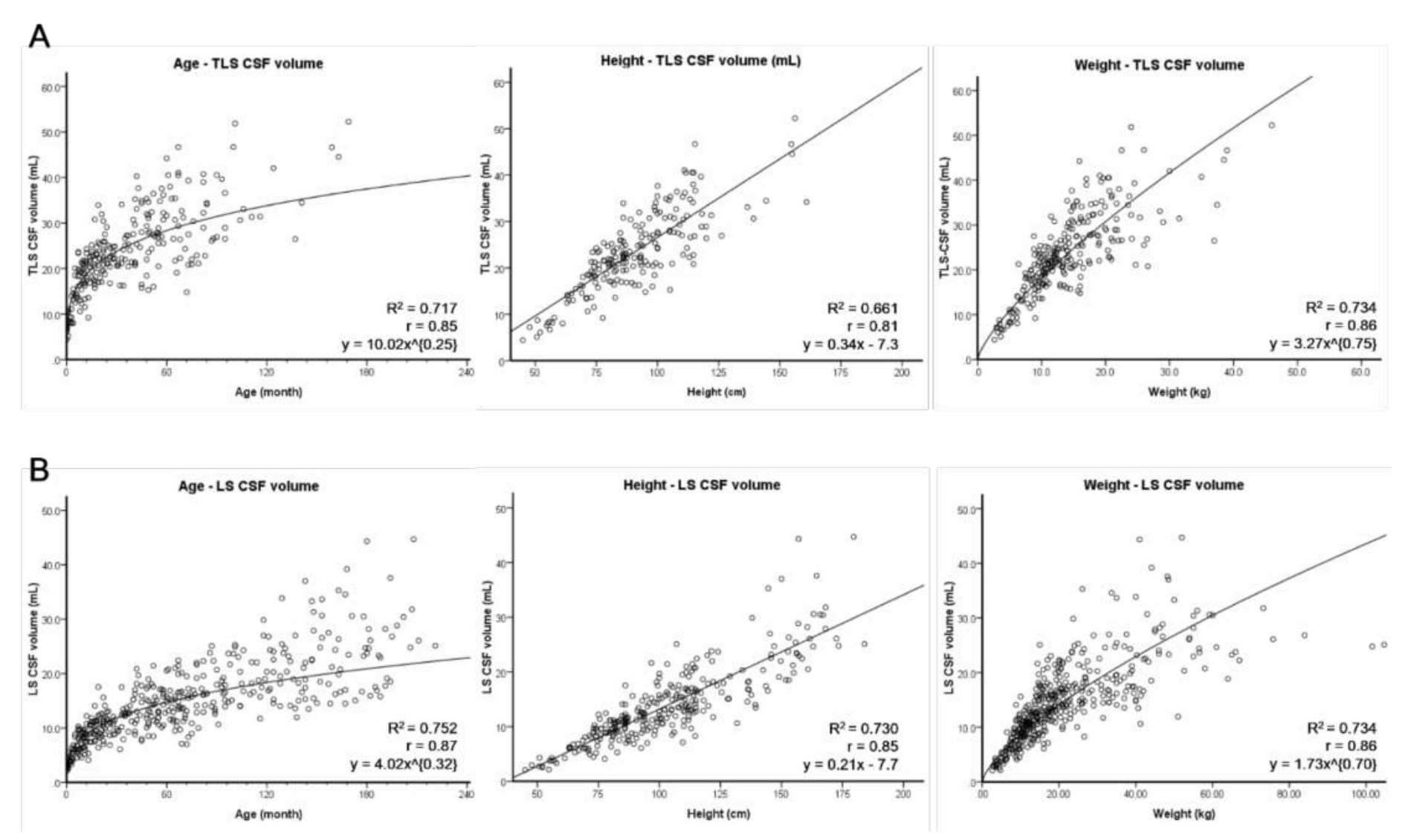

Figure 2. Measurement of CSF area by subtracting the spinal cord area from the dural sac area at level T12 in both T1 MR image (A) and T2 MR image (B)

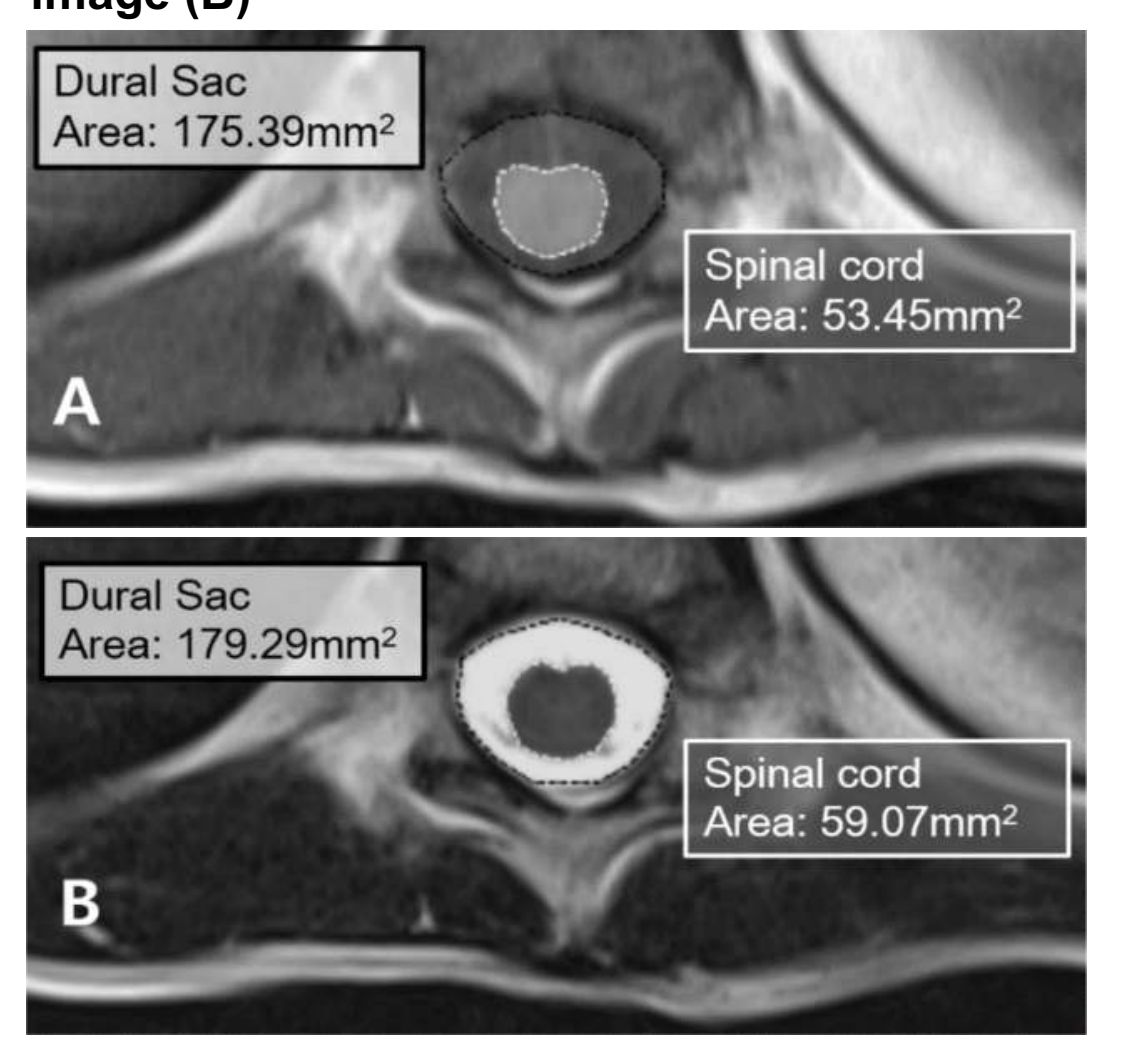

